Rebekka Bratschi / Astrid Hirzel / Céline Mavrot / Markus Nussbaumer / Lucas Stutz / Felix Uhlmann

\title{
Literaturhinweise - Repères bibliographiques - Riferimenti bibliografici - Indicaziuns bibliograficas
}

Beitragsart: Literaturhinweise 
Rebekka Bratschi / Astrid Hirzel / Céline Mavrot / Markus Nussbaumer / Lucas Stutz / Felix Uhlmann, Literaturhinweise - Repères bibliographiques - Riferimenti bibliografici - Indicaziuns bibliograficas, in:

LeGes 31 (2020) 1

\section{Inhaltsübersicht}

1. Recht und Rechtssoziologie

1.1. Rechts- und Gesetzgebungstheorie

1.2. Gesetzgebungstechnik und -methodik

1.3. Organisation und Verfahren der Rechtsetzung

1.4. Rechtssoziologie und Rechtspolitik

1.5. Europarecht und europäische Integration

1.6. Verfassungsrecht

1.7. Einzelfragen

2. Sprachwissenschaft und Rechtslinguistik

2.1. Rechts- und Verwaltungssprache

2.2. Verständlichkeitsforschung

2.3. Mehrsprachigkeit, Übersetzung

2.4. Einzelfragen

2.5. Hilfsmittel

3. Evaluation

3.1. Evaluationsansätze und -methoden

3.2. Evaluationspraxis

3.3. Evaluation in Politik und Verwaltung

3.4. Evaluationsstudien

3.5. Evaluationsfachlichkeit

\section{Recht und Rechtssoziologie}

\subsection{Rechts- und Gesetzgebungstheorie}

Christodoulidis, Emilios / Dukes, Ruth / Goldoni, Marco (Hrsg.) (2019): Research handbook on critical legal theory, Cheltenham.

Filbinger, Konstantin (2019): Staatliches Steuerungsselbstvertrauen. Definition, Manifestation, Vergleich, Hamburg.

HaAck, Stefan (2019): Theorie des öffentlichen Rechts, 2 Bände, Tübingen.

HäNNI, Julia (2019): Rechtsphilosophie in a nutshell, Zürich.

Hey, Johanna / Schwarz, Kyrill-Alexander / Reimer, Ekkehart / Karpen, Ulrich / Kirchhof, FerdinAND (2019): Chancen guter Gesetzgebung in einer komplexen Welt. Redemanuskripte zur Tagung am 21. und 22. November 2018, Berlin.

Hilbert, Patrick / Rauber, Jochen (Hrsg.) (2019): Warum befolgen wir Recht? Rechtsverbindlichkeit und Rechtsbefolgung in interdisziplinärer Perspektive, Tübingen.

Honsell, Heinrich (2019): Was ist Gerechtigkeit?, Bern.

Kirste, Stephan (2019): Einführung in die Rechtsphilosophie, Baden-Baden.

Koch, Felix / Mohseni, Amir / Schweikard, David P. (Hrsg.) (2019): Analytische Rechtsphilosophie. Grundlagentexte, Berlin.

Kramer, Ernst A. (2019): Juristische Methodenlehre, 6. Aufl., Bern. 
Rebekka Bratschi / Astrid Hirzel / Céline Mavrot / Markus Nussbaumer / Lucas Stutz / Felix Uhlmann, Literaturhinweise - Repères bibliographiques - Riferimenti bibliografici - Indicaziuns bibliograficas, in: LeGes 31 (2020) 1

Lege, Joachim (Hrsg.) (2019): Gelingendes Recht. Über die ästhetische Dimension des Rechts, Tübingen.

Mahlmann, Matthias (2019): Konkrete Gerechtigkeit. Eine Einführung in Recht und Rechtswissenschaft der Gegenwart, 4. Aufl., Baden-Baden.

Mahlmann, Matthias (2019): Rechtsphilosophie und Rechtstheorie, 5. Aufl., Baden-Baden.

Mayer-Maly, Theo / Honsell, Heinrich / Mayer-Maly, Dorothea (Hrsg.) (2019): Recht - Gerechtigkeit - Rechtswissenschaft. Gesammelte Schriften von Theo Mayer-Maly, Wien.

Möllers, Thomas M.J. (2019): Juristische Methodenlehre, 2. Aufl., München.

Muthorst, Olaf (2019): Grundlagen der Rechtswissenschaft. Methode - Begriff - System, 2. Aufl. München.

Potacs, Michael (2019): Rechtstheorie, 2. Aufl., Wien.

Philippopoulos-Mihalopoulos, Andreas (Hrsg.) (2019): Routledge handbook of law and theory, London.

Seelmann, Kurt / Demko, Daniela (2019): Rechtsphilosophie, 7. Aufl., München.

Uhlmann, Felix (Hrsg.) (2019): Verhältnismässigkeit als Grundsatz in der Rechtsetzung und Rechtsanwendung. 17. Jahrestagung des Zentrums für Rechtsetzungslehre, Zürich.

Uhlmann, Felix / Bukovac, Jasmina (2019): Das Verhältnismässigkeitsprinzip aus dem Blickwinkel der Rechtsetzungslehre, in: Felix Uhlmann (Hrsg.), Verhältnismässigkeit als Grundsatz in der Rechtsetzung und Rechtsanwendung. 17. Jahrestagung des Zentrums für Rechtsetzungslehre, Zürich, S. 33 ff.

Weber-Grellet, Heinrich (2019): Rechtsphilosophie und Rechtstheorie, 7. Aufl., Münster.

Wyss, Martin (2019): Rechtsetzungsverantwortung zwischen Vertrauen, Kontrolle und Mängelrügen, in: Parlament, Heft 3, S. 17 ff.

\subsection{Gesetzgebungstechnik und -methodik}

Bundesamt für Justiz (Hrsg.) (2019): Gesetzgebungsleitfaden. Leitfaden für die Ausarbeitung von Erlassen des Bundes, Bern.

Dévaud, Blaise / Hugentobler, Wolfgang (2019): Automatisierung normativer Prozesse am Beispiel von «stadtrecht.bern.ch», in: LeGes 30 (2019) 3.

FlüCKiger, Alexandre (2019): L'impact de l'intelligence artificielle sur l'écriture des lois: du code de lois à la loi encodée, in: LeGes 30 (2019) 3.

Flückiger, Alexandre (2019): (Re)faire la loi. Traité de légistique à l'ère du droit souple, Berne.

Müller, Georg (2019): Methode und Verfahren der Verhältnismässigkeitsprüfung in der Gesetzgebung, insbesondere Prognose-, Abwägungs-, Beobachtungs- und Nachbesserungspflichten, in: 
Rebekka Bratschi / Astrid Hirzel / Céline Mavrot / Markus Nussbaumer / Lucas Stutz / Felix Uhlmann, Literaturhinweise - Repères bibliographiques - Riferimenti bibliografici - Indicaziuns bibliograficas, in: LeGes 31 (2020) 1

Felix Uhlmann (Hrsg.), Verhältnismässigkeit als Grundsatz in der Rechtsetzung und Rechtsanwendung. 17. Jahrestagung des Zentrums für Rechtsetzungslehre, Zürich, S. $101 \mathrm{ff}$.

Vontobel-Lareida, Eva (2019): Umsetzung der Verhältnismässigkeitsprüfung im Gesetzes-und Verordnungsrecht, in: Felix Uhlmann (Hrsg.), Verhältnismässigkeit als Grundsatz in der Rechtsetzung und Rechtsanwendung. 17. Jahrestagung des Zentrums für Rechtsetzungslehre, Zürich, S. $57 \mathrm{ff}$.

\subsection{Organisation und Verfahren der Rechtsetzung}

DANKowski, Anne (2019): Expertengruppen in der europäischen Rechtsetzung. Sachverständige Beratung der Europäischen Kommission im Spannungsfeld von Funktionalität und Gemeinwohlorientierung - eine Untersuchung des Regelungsrahmens, Berlin.

FuHrer, Corina (2019): Die Umsetzung kantonaler Volksinitiativen, Zürich.

Graf, Martin (2019): Das Verordnungsveto - ein Instrument zur Wahrung der Rechtsetzungsprärogative des Parlaments, in: Parlament, Heft 3, S. $10 \mathrm{ff}$.

Hodel, Peter (2019): Sollen Parlamente auf die Verordnungsgebung der Regierung Einfluss nehmen? Erfahrungen aus dem Kanton Solothurn, in: Parlament, Heft 3, S. 3 f.

Musliu, Nagihan (2019): Die Umsetzung eidgenössischer Volksinitiativen, Zürich.

Müller, Georg (2019): Sollen Parlamente auf die Verordnungsgebung der Regierung Einfluss nehmen? Thesen mit Kommentar, in: Parlament, Heft 3, S. 5 ff.

Popelier, Patricia / Xanthaki, Helen / Robinson, William / Tiago Silveira, João / Uhlmann, Felix (Hrsg.) (2019): Lawmaking in multi-level settings. Legislative challenges in federal systems and the European Union, Baden-Baden.

Rast, Florian / Wilhelm, Christian (2019): Mehr Gesetzgebung in die Lehre! Die LMU-Gesetzgebungswerkstatt, in: Zeitschrift für Gesetzgebung 34/2, S. 182-189.

\subsection{Rechtssoziologie und Rechtspolitik}

Bucher Nevin, Martina, Jugend und Politik (2019): Das Jugendvorstossrecht in den Gemeinden, Zürich.

Dal Molin-Kränzlin, Alexandra / Schneuwly, Anne Mirjam / Stojanovic, Jasna (Hrsg.) (2019): Digitalisierung, Gesellschaft, Recht. Analysen und Perspektiven von Assistierenden des Rechtswissenschaftlichen Instituts der Universität Zürich, Zürich.

Hayden, Karen E. (2019): Society and law, Lanham.

Meister-Scheufelen, Gisela (2018): Schuldet der Gesetzgeber ein gutes Gesetz?, in: Zeitschrift für Gesetzgebung 33/3, S. 231-240. 
Rebekka Bratschi / Astrid Hirzel / Céline Mavrot / Markus Nussbaumer / Lucas Stutz / Felix Uhlmann, Literaturhinweise - Repères bibliographiques - Riferimenti bibliografici - Indicaziuns bibliograficas, in: LeGes 31 (2020) 1

Strohmeier, Rudolf W. / Gamisch, Astrid (2019): Die Veröffentlichung von Gesetzen im digitalen Zeitalter, in: Die öffentliche Verwaltung, Heft 12.

Tejani, Riaz (2019): Law and society today, Oakland.

\subsection{Europarecht und europäische Integration}

Besson, Samantha (2019) : Droit constitutionnel européen, Bern.

Bieber, Roland / Epiney, Astrid / HaAg, Marcel / Kotzur, Markus (2019): Europarecht in Fragen und Antworten, 5. Aufl., Baden-Baden.

Bieber, Roland / Epiney, Astrid / Haag, Marcel / Kotzur, Markus (2019): Die Europäische Union, Europarecht und Politik, 13. Aufl., Baden-Baden.

Bieber, Roland (2019): Europarecht. Textausgabe mit einer Einführung von Prof. Dr. Roland Bieber, begründet durch Prof. Dr. Hans-Joachim Glaesner, 25. Aufl., Baden-Baden.

Brun, Felix (2019): Sprechen wir über Europa. Markante Reden und Texte aus 100 Jahren, Baden.

Epiney, Astrid / Mosters, Robert / Rieder, Andreas (2019): Europarecht, 2 Bände, 4. Aufl., Bern.

Epiney, Astrid / Hehemann, Lena / Zltescu, Petru Emanuel (Hrsg.) (2019): Schweizerisches Jahrbuch für Europarecht, 2018/2019, Bern.

Fischer, Kristian / Fetzer, Thomas (2019): Europarecht, 12. Aufl., Heidelberg.

Fischer, Kristian / Fetzer, Thomas (2019): Fälle zum Europarecht, 9. Aufl., Heidelberg.

Hafner, Gerhard / Kumin, Andreas / Weiss, Friedl (Hrsg.) (2019): Recht der Europäischen Union, 2. Aufl., Wien.

Hanstein, Dennis F. (2019): Subsidiarität und die demokratische Legitimation europäischer Legislativakte, Baden-Baden.

Herdegen, Matthias (2019): Europarecht, 21. Aufl., München.

Kellerhals, Andreas (Hrsg.) (2019): Europa in der Welt. Referate zu Fragen der Zukunft Europas 2018, Zürich.

Kellerhals, Andreas / Baumgartner, Tobias (Hrsg.) (2019): Wirtschaftsrecht Schweiz - EU. Überblick und Kommentar 2018/19, Zürich.

López Hormigo, Sonia (2019): Die Auswirkungen der Charta der Grundrechte auf die Verfassungsgerichtsbarkeit in der Europäischen Union und in den Mitgliedstaaten, Zürich.

Oesch, Matthias (2019): Europarecht. Band 1: Grundlagen, Institutionen, Verhältnis SchweizEU, 2. Aufl., Bern. 
Rebekka Bratschi / Astrid Hirzel / Céline Mavrot / Markus Nussbaumer / Lucas Stutz / Felix Uhlmann, Literaturhinweise - Repères bibliographiques - Riferimenti bibliografici - Indicaziuns bibliograficas, in: LeGes 31 (2020) 1

\subsection{Verfassungsrecht}

Biaggini, Giovanni / Ehrenzeller, Bernhard (Hrsg.) (2019): Textausgabe Öffentliches Recht, 9. Aufl., Zürich.

Donath, Philipp B. (Hrsg.) (2019): Verfassungen - ihre Rolle im Wandel der Zeit. 59. Assistententagung Öffentliches Recht, Frankfurt am Main 2019, Baden-Baden.

Giger, Hans (2019): Verfassung auf dem Prüfstand. Gesetzgebung im Fokus allgemeiner Rechtsprinzipien, Bern.

Hottelier, Michel / Hertig Randall, Maya / Flückiger, Alexandre (Hrsg.) (2019) : Etudes en l'honneur du Professeur Thierry Tanquerel, entre droit constitutionnel et droit administratif, questions autour du droit de l'action publique, Genf.

JaAg, Tobias / Rüssli, Markus, (2019): Staats- und Verwaltungsrecht des Kantons Zürich, 5. Aufl., Zürich.

Tschentscher, Axel / Lienhard, Andreas / Sprecher, Franziska (2019): Öffentliches Recht, Verfassungsrecht, Verwaltungsrecht, öffentliches Verfahrensrecht, 2. Aufl., Zürich.

Waldmann, Bernhard / Wiederkehr, René (2019): Allgemeines Verwaltungsrecht, Zürich.

\subsection{Einzelfragen}

Ambühl, Michael / Scherer, Daniela S. (2019), Zum Entwurf des Institutionellen Abkommens, in: Jusletter vom 4. Februar 2019.

Auer, Christoph / Müller, Markus / Schindler, Benjamin (Hrsg.) (2019): VwVG, Bundesgesetz über das Verwaltungsverfahren. Kommentar, 2. Aufl., Zürich.

Bachmann, Gregor (2019): Anspruch auf Verfahren und Entscheid. Der Zugang zum Verwaltungsverfahren und zur Verwaltungsrechtspflege unter besonderer Berücksichtigung der verfassungsrechtlichen Verfahrensgarantien, Bern.

Baldi, Marino (2019): Zu den Auswirkungen des geplanten Rahmenabkommens mit der EU, in: Jusletter vom 18. März 2019.

Beaucamp, Guy / Beaucamp, Jаков (2019): Methoden und Technik der Rechtsanwendung, 4. Aufl., Heidelberg.

Breitenband, Detlef (2019): Konsens - der Grund der Legitimität. Studie zu Kants und Habermas' Theorien der Legitimation des Rechts, Wiesbaden.

Coradi, Christina (2019): Max Imboden, Pionier des schweizerischen Staats- und Verwaltungsrechts, in: Aktuelle juristische Praxis, Heft 4, S. 470 ff.

Doleschal, Ralph David (2019): Die abstrakte Normenkontrolle in den Kantonen, Zürich.

Epiney, Astrid / Affolter, Sian (2019): Das Institutionelle Abkommen und die Unionsbürgerrichtlinie, in: Jusletter vom 11. März 2019. 
Rebekka Bratschi / Astrid Hirzel / Céline Mavrot / Markus Nussbaumer / Lucas Stutz / Felix Uhlmann, Literaturhinweise - Repères bibliographiques - Riferimenti bibliografici - Indicaziuns bibliograficas, in: LeGes 31 (2020) 1

Epiney, Astrid / Frei, Nula (2019): Zur Übernahme weiterentwickelten EU-Rechts durch den Bundesrat, in: Jusletter vom 12. August 2019.

Epiney, Astrid / Hehemann, Lena (2019): Die Tragweite des Institutionellen Abkommens im Bereich der Arbeitnehmerentsendung, in: Jusletter vom 8. April 2019.

Fleischmann, Florian / Boxler, Adrian (2019): Übungsbuch Verwaltungsrecht. Repetitionsfragen, Übungsfälle und bundesgerichtliche Leitentscheide, 3. Aufl., Zürich.

Flückiger, Alexandre (2019) : (Re)faire la loi. Traité de légistique à l'ère du droit souple, Bern.

Foglia, Aldo (2019): Per discutere la civica. $2^{\mathrm{a}}$ ed. con una postfazione di Pio Caroni, Manno.

GaL, Jens (2019): Ta pathetika prooimia test Europes. Zu Sinn und Unsinn der Erwägungsgründe, in: Myops 13/37, S. 4-18.

Geerlings, Jörg (2019): Die Akzeptanz europäischen Rechts aus deutscher Perspektive, in: Die öffentliche Verwaltung, Heft 5.

Giersdorf, Fabian (2019): Der informelle Trilog. Das Schattengesetzgebungsverfahren der Europäischen Union, Berlin.

Gruber, Daniel (2019): Law without authority or limits. Kelsen's dilemma, Hannover.

Häner, Isabelle / Waldmann, Bernhard (Hrsg.) (2019): 7. Forum für Verwaltungsrecht. Staatliche Aufsicht über die Wirtschaft und ihre Akteure, Bern.

Haux, Dario Henri / Picecchi, Dario / Schreiber, Markus (Hrsg.) (2019): Recht und Risiko. Junge Rechtswissenschaft Luzern, Zürich.

Hofstetter, David (2019): Schematisierungen und Verhältnismässigkeit, in: Felix Uhlmann (Hrsg.), Verhältnismässigkeit als Grundsatz in der Rechtsetzung und Rechtsanwendung, 17. Jahrestagung des Zentrums für Rechtsetzungslehre, Zürich, S. 87 ff.

Hong, Mathias (2019): Abwägungsfeste Rechte. Von Alexys Prinzipien zum Modell der Grundsatznormen, Tübingen.

HoRst, Johan (2019): Transnationale Rechtserzeugung. Elemente einer normativen Theorie der Lex Financiaria, Tübingen.

Keller Läubli, Lucy (2019): Die sogenannte Lex Handschlag des Kantons Basel-Landschaft unter Aspekten guter Gesetzgebung, in: LeGes 30 (2019) 1.

Kipker, Dennis-Kenji / Kopp, Matthias / Wiersbinski, Peter / Marschelke, Jan-Christoph / Hamann, Falk / Weichold, Martin (Hrsg.) (2019): Der normative Druck des Faktischen. Tagungen des Jungen Forums Rechtsphilosophie (JFR) in der Internationalen Vereinigung für Rechts- und Sozialphilosophie (IVR) im September 2016 in Bremen und im September 2017 in Regensburg, Stuttgart.

KLosko, George (2019): Why should we obey the law?, Cambridge.

Lambelet, Jean-Christian (2019): De la démocratie directe en Suisse, son cadre, sa pratique, une analyse statistique et une évaluation sur le fond, Genf. 
Rebekka Bratschi / Astrid Hirzel / Céline Mavrot / Markus Nussbaumer / Lucas Stutz / Felix Uhlmann, Literaturhinweise - Repères bibliographiques - Riferimenti bibliografici - Indicaziuns bibliograficas, in: LeGes 31 (2020) 1

Legnini, Giovanni / Piccione, Daniele (2019): I poteri pubblici nell'età del disincanto. L'unità perduta tra legislazione, regolazione e giurisdizione, Roma.

Lendi Martin (2019): Freude am Recht, Belebung des Rechts, der Rechtsordnungen, des Rechtsstaates, der Demokratie, Zürich.

Mächler, August (2019): Verhältnismässiger Einsatz von Regelungsinstrumenten, in: Felix Uhlmann (Hrsg.), Verhältnismässigkeit als Grundsatz in der Rechtsetzung und Rechtsanwendung, 17. Jahrestagung des Zentrums für Rechtsetzungslehre, Zürich, S. 67 ff.

Mathis, Klaus (2019): Effizienz statt Gerechtigkeit? Auf der Suche nach den philosophischen Grundlagen der ökonomischen Analyse des Rechts, 4. Aufl., Berlin.

Meinel, Florian (2019): Selbstorganisation des parlamentarischen Regierungssystems. Vergleichende Studien zu einem Verfassungsproblem der Bundesrepublik Deutschland, Tübingen.

Müller, Markus (2019): Verhältnismässigkeit. Ein Verfassungsprinzip, zwischen Rechtsregel und Metaregel, in: Felix Uhlmann (Hrsg.), Verhältnismässigkeit als Grundsatz in der Rechtsetzung und Rechtsanwendung, 17. Jahrestagung des Zentrums für Rechtsetzungslehre, Zürich, S. 7 ff.

Norgueira de Brito, Miguel / Herdy, Rachel / Damele, Giovanni / Moniu Lopes, Pedro / Silva Sampaio, Jorge (Hrsg.) (2019): The Role of Legal Argumentation and Human Dignity in Constitutional Courts. Proceedings of the Special Workshops held at the 28th World Congress of the International Association for Philosophy of Law and Social Philosophy in Lisbon, 2017, Stuttgart.

Notter, Markus (2019): Institutionelles Rahmenabkommen - ein europapolitischer Zwischenruf, in: Zeitschrift für Europarecht, Heft 1, S. 4 ff.

Pelegrino da Silva, Matheus (2019): Kelsens Theorie der Rechtserkenntnis. Zugleich eine kritische Betrachtung der Positivität als Eigenschaft des Rechts, Berlin.

Puppe, Ingeborg (2019): Kleine Schule des juristischen Denkens, 4. Aufl., Stuttgart.

Rast, Florian / Wilhelm, Christian (2019): Mehr Gesetzgebung in die Lehre! - Die LMU-Gesetzgebungswerkstatt, in: Zeitschrift für Gesetzgebung, Heft 2, S. 182-189.

Reich, Johannes (2019): Switzerland. The «Globalisation Paradox» in Constitutional Practice, in: Albert Richard / David Landau / Pietro Faraguna / Simon Drugda (Hrsg.), 2018 Global Review of Constitutional Law, Boston.

Ritz, Adrian / Haldemann, Theo / Sager, Fritz (Hrsg.) (2019): Blackbox Exekutive. Regierungslehre in der Schweiz, Basel.

Schindler, Benjamin (2019): «Von Jahr zu Jahr werden die Gesetze komplizierter.», in: Schweizerisches Zentralblatt für Staats- und Verwaltungsrecht, Heft 5, S. 233 f.

SVVOR, Schweizerische Vereinigung für Verwaltungsorganisationsrecht (2019): Verwaltungsorganisationsrecht - Staatshaftungsrecht - öffentliches Dienstrecht / Droit public de l'organisation - responsabilité des collectivités publiques - fonction publique, Jahrbuch 2018/2019 - Annuaire 2018/2019 (deutsch/französisch), Bern. 
Rebekka Bratschi / Astrid Hirzel / Céline Mavrot / Markus Nussbaumer / Lucas Stutz / Felix Uhlmann, Literaturhinweise - Repères bibliographiques - Riferimenti bibliografici - Indicaziuns bibliograficas, in: LeGes 31 (2020) 1

Uhlmann, Felix (Hrsg.) (2019): Verhältnismässigkeit als Grundsatz in der Rechtsetzung und Rechtsanwendung. 17. Jahrestagung des Zentrums für Rechtsetzungslehre. 1. Aufl., Zürich.

Vetter, Sarah (2019): Die Schweiz in ihrer Beziehung zur EU. Ein Vorbild für das zukünftige Verhältnis Grossbritanniens zur EU infolge des Brexit?, Hamburg.

Von Heydebrand, Sibylle (2019): Stimmrecht und kantonale Autonomie. Allgemeines und gleiches Stimmrecht am Beispiel der Nordwestschweizer Kantone, Basel.

Voss, Henning (2019): Unternehmenswissen als Regulierungsressource. Der aufsichtsrechtliche Zugriff auf bankinterne Strukturen, Tübingen.

Wimmer, Andreas W. (2019): Rechtsverhältnisse im öffentlichen Recht. Ein Perspektivenwechsel, Wien.

Wyss, Martin Philipp (2018): Grauzonen der Rechtsetzung -- Von Weisungen, Rundschreiben und anderen Erscheinungen, in: LeGes 29 (2018) 3.

Zeccola, Marc (2019): Die Akzeptanz im Verwaltungsverfahren - Ein Beitrag zur Aufwertung der Akzeptanz als Rechtmässigkeitsvoraussetzung, In: Die öffentliche Verwaltung, Heft 3.

\section{Sprachwissenschaft und Rechtslinguistik}

\subsection{Rechts- und Verwaltungssprache}

AdamziK, Kirsten (2018): «Cyberadministration»vs. «E-Government». Zur Sprache der Schweizer Bundesverwaltung im Spiegel ihrer Hilfsmittel zur Textgestaltung, in: Thomas Tinnefeld (Hrsg.): Fachsprache Französisch. Facettenreich, faszinierend, funktional, Saarbrücken, S. 109137.

Baumann, Klaus-Dieter (2018): Die fachkommunikativen Grundlagen der Verwaltungssprache. Eine Annäherung an die interdisziplinäre Betrachtung von Fachlichkeit, in: Lenka Vaňková (Hrsg.): Fachlichkeit und Fachsprachlichkeit in varianten Kontexten, Berlin, S. 35-47.

Behschnitt, Miriam (2019): Die Fachtextsorte Gesetz. Eine kontrastive stilistische Untersuchung anhand des deutschen Aufenthaltsgesetzes und britischer Immigration Acts, Berlin.

Bendix, Ludwig (2018/1904): Terminologie und Begriffsbildung im Gesetzentwurf über den Versicherungs-Vertrag, Reprint 2018, Berlin/Boston.

Bezemek, Christoph (2018): Die Sprache der Bürokratie als Sprache der Folgerichtigkeit. Ein Essay, in: Journal für Rechtspolitik 165/3, S. 165-171.

Broekman, JAN M. (2019): Rethinking law and language, the flagship «speech», Cheltenham.

Bruno, Giovanni (2019): L'aggettivo «sabatale»: un neologismo incipiente nel linguaggio burocratico-amministrativo?, in: LeGes 30 (2019) 3.

Burger, Simon (Hrsg.) (2019): Recht und Sprache. Tagung der Österreichischen Assistentinnen und Assistenten Öffentliches Recht, Salzburg 2018, Wien. 
Rebekka Bratschi / Astrid Hirzel / Céline Mavrot / Markus Nussbaumer / Lucas Stutz / Felix Uhlmann, Literaturhinweise - Repères bibliographiques - Riferimenti bibliografici - Indicaziuns bibliograficas, in: LeGes 31 (2020) 1

Bydlinski, Peter (2019): Über den Versuch einer vor allem sprachlichen Verbesserung des österreichischen ABGB, in: LeGes 30 (2019) 1.

Cattani, Paola / Sergio, Giuseppe (a c. di) (2018): Comunicare cittadinanza nell'era digitale. Saggi sul linguaggio burocratico 2.0, Milano.

Cavagnoli-Woelk, Stefania / Mori, Laura (2019): Gender in legislative languages. From EU to national law in English, French, German, Italian and Spanish, Berlin.

Egger, Jean-Luc (2019): A norma di (chi) legge. Peculiarità dell'italiano federale, Milano.

Flückiger, Alexandre (Ed) (2019): La rédaction administrative et législative inclusive. La francophonie entre impulsions et résistances, Berne.

Forowicz, Magdalena (2019): Les limites de la visualisation juridique en matière de réglementation, in: LeGes 30 (2019) 3.

Höfler, Stefan (2019): Müssen oder nicht müssen? Die Modalität von Rechtssätzen aus redaktioneller Sicht, in: LeGes 30 (2019) 2.

Humbley, John / Budin, Gerhard / Laurén, Christer (Eds) (2018): Languages for Special Purposes. An International Handbook, Berlin.

Kaplan, JefFrey P. (2020): Linguistics and law, London.

Mattila, Heikкi (2018): Legal language, in: John Humbley / Gerhard Budin / Christer Laurén (Eds): Languages for Special Purposes. An International Handbook, p. 113-150.

Nussbaumer, Markus (2019): «Alle Schweizer sind vor dem Gesetze gleich». Anekdotisches Nachwort zum Gendern in Gesetzen, in: LeGes 30 (2019) 2.

Parlament der Deutschsprachigen Gemeinschaft Belgiens (Hrsg.) (2019): Nationale Variation in der deutschen Rechtsterminologie. Beiträge zur Tagung vom 12. und 13. Oktober 2018 im Seminarzentrum Kloster Heidelberg in Eupen, Eupen.

Ryan, Richard (2018): Controlled Language, in: John Humbley / Gerhard Budin / Christer Laurén (Eds): Languages for Special Purposes. An International Handbook, p. 289-306.

Sandrini, Peter (2018): Terminological work in different domains: legal terminology, in: John Humbley / Gerhard Budin / Christer Laurén (Eds): Languages for Special Purposes. An International Handbook, p. 522-534.

Schindler, Benjamin (2019): «Von Jahr zu Jahr werden die Gesetze komplizierter.», in: Schweizerisches Zentralblatt für Staats- und Verwaltungsrecht 120, S. $233 \mathrm{f}$.

Schmidt-KöNig, Christine (2019), Introduction à la langue juridique française, 4. Aufl., BadenBaden.

Skocze, Izabela (2019): Implicatures within legal language, Cham.

Tirlilë, Ulla (2018): Legal discourse as an example of domain specific science, in: John Humbley / Gerhard Budin / Christer Laurén (Eds): Languages for Special Purposes. An International Handbook, p. 581-405. 
Rebekka Bratschi / Astrid Hirzel / Céline Mavrot / Markus Nussbaumer / Lucas Stutz / Felix Uhlmann, Literaturhinweise - Repères bibliographiques - Riferimenti bibliografici - Indicaziuns bibliograficas, in: LeGes 31 (2020) 1

Vaňková, Lenka (Hrsg.) (2018): Fachlichkeit und Fachsprachlichkeit in varianten Kontexten, Berlin.

Vellutino, Daniela (2018): L'italiano istituzionale per la comunicazione pubblica, Bologna.

Vogel, Friedemann (Hrsg.) (2019): Legal Linguistics Beyond Borders: Language and Law in a World of Media, Globalisation and Social Conflicts. Relaunching the International Language and Law Association (ILLA), Berlin.

\subsection{Verständlichkeitsforschung}

Bartz, Thomas (2018): Texte sprachbewusst optimieren. Ein linguistisches Kategoriensystem für die computergestützte Revision qualitätsrelevanter sprachlicher Merkmale in Texten, Berlin.

Bock, Bettina M. (2019): «Leichte Sprache» - Kein Regelwerk. Sprachwissenschaftliche Ergebnisse und Praxisempfehlungen aus dem LeiSA-Projekt, Berlin.

Bydlinski, Peter (2019): Über den Versuch einer vor allem sprachlichen Verbesserung des österreichischen $A B G B$, in: LeGes 30 (2019) 1.

Engberg, Jan / Luttermann, Karin / Cacchiani, Silvia / Preite, Chiara (Hrsg.) (2018): Popularization and knowledge mediation in the law. Popularisierung und Wissensvermittlung im Recht, Berlin/Münster.

Forowicz, Magdalena (2019): Les limites de la visualisation juridique en matière de réglementation, in: LeGes 30 (2019) 3.

Hallik, Sibylle (2019): Die Sprachberatung des Redaktionsstabs der GfdS beim Deutschen Bundestag, in: Der Sprachdienst 63/5-6, S. 192-199.

Höfler, Stefan (2019): Die Sprache des Gesetzes soll verständlich sein, in: Plädoyer 4, S. 42-46.

Höfler, Stefan (2019): Making the Law More Transparent. Text Linguistics for Legislative Drafting, in: Friedemann Vogel (Ed): Legal Linguistics Beyond Borders: Language and Law in a World of Media, Globalisation and Social Conflicts. Relaunching the International Language and Law Association (ILLA), p. 229-252.

Junod, Simon (2019): La rédaction législative et administrative inclusive, in: LeGes 30 (2019) 1.

Li, Shuangling (2019): Communicative Significance of Vague Language: A Diachronic Corpusbased Study of Legislative Texts, in: English for Specific Purposes 53, p. 104-117.

Wagner, Wendy / Walker, Will (2019): Incomprehensible! A Study of How Our Legal System Encourages Incomprehensibility, Why it Matters, and What We Can Do About It, Cambridge.

\subsection{Mehrsprachigkeit, Übersetzung}

Biel, Łucja / Engberg, Jan / Ruano, Rosario Martín / Sosoni, Vilelmini (Eds) (2019): Research Methods in Legal Translation and Interpreting: Crossing Methodological Boundaries, London. 
Rebekka Bratschi / Astrid Hirzel / Céline Mavrot / Markus Nussbaumer / Lucas Stutz / Felix Uhlmann, Literaturhinweise - Repères bibliographiques - Riferimenti bibliografici - Indicaziuns bibliograficas, in: LeGes 31 (2020) 1

Biel, Łucja / Koźbiat, Dariusz / Wasilewska, Katarzyna (2019): The formulaicity of translations across EU institutional genres. A corpus-driven analysis of lexical bundles in translated and non-translated language, in: Translation Spaces 8/1, p. 67-92.

Chartier-Brun, Pascale (2018): Translating legislative documents at the European Parliament: e-Parliament, XML, SPA and the Cat4Trad workflow, in: Zeitschrift für Europäische Rechtslinguistik.

Chartier-Brun, Pascale / Mahler, Katharina (2018): Machine Translation and Neural Networks for a Multilingual EU, Zeitschrift für Europäische Rechtslinguistik.

Colneric, Ninon (2019): Multilingual and Supranational Law in the EU: «United in Diversity» or «Tower of Babel»?, in: Friedemann Vogel (Ed): Legal Linguistics Beyond Borders: Language and Law in a World of Media, Globalisation and Social Conflicts. Relaunching the International Language and Law Association (ILLA), p. 167-186.

Cross-cutting Methodological Issues in Legal Translation (2019): Special issue of: Comparative Legilinguistics. International Journal for Legal Communication 37.

Evangelista, Daria (2019): La formulazione del diritto mite: l'esempio dell'Accordo di Parigi sul clima, in: LeGes 30 (2019) 2.

Felici, Annarita / Griebel, Cornelia (2019): The challenge of multilingual "plain language» in translation-mediated Swiss administrative communication. A preliminary comparative analysis of insurance leaflets, in: Translation Spaces 8/1, p. 167-191.

GArré, Roy (2019): Un solo diritto, più lingue: il multilinguismo interpretativo elvetico, in: LeGes 30 (2019) 3.

Mattissen, Johanna (2019): Coordination, ambiguity and divergence in legal acts of the European Union, in: Zeitschrift für Europäische Rechtslinguistik.

Meier, Niklaus (2019): Terminologie juridique allemande, 3. Aufl., Basel.

Mori, Laura (Ed) (2018): Observing Eurolects. Corpus analysis of linguistic variation in EU law, Amsterdam/Philadelphia.

Nehls, Benedikt (2019): Die Auslegung mehrsprachiger völkerrechtlicher Verträge. Eine Darstellung der Auslegungsregeln unter Berücksichtigung ihrer historischen Entwicklung, Berlin.

Peruzzo, Katia (2019): When international case-law meets national law. A corpus-based study on Italian system-bound loan words in ECtHR judgments, in: Translation Spaces 8/1, p. 12-38.

Pingel, Isabelle (2019): Le multilinguisme à la Cour de justice de l'Union européenne: questions choisies, in: International Journal for the Semiotics of Law 32/2, p. 449-462.

Prieto Ramos, Fernando (Ed) 2019: Corpus-Based Research in Legal and Institutional Translation. Special issue of: Translation Spaces 8/1.

Prieto Ramos, Fernando, 2019, The use of corpora in legal and institutional translation studies. Directions and applications, in: Translation Spaces 8/1, p. 1-11. 
Rebekka Bratschi / Astrid Hirzel / Céline Mavrot / Markus Nussbaumer / Lucas Stutz / Felix Uhlmann, Literaturhinweise - Repères bibliographiques - Riferimenti bibliografici - Indicaziuns bibliograficas, in: LeGes 31 (2020) 1

Prieto Ramos, Fernando / Cerutti, Giorgina / Guzmán, Diego (2019): Building representative multi-genre corpora for legal and institutional translation research. The LETRINT approach to text categorization and stratified sampling, in: Translation Spaces 8/1, p. 93-116.

SAndrini, Peter (2018): Legal translation, in: John Humbley / Gerhard Budin / Christer Laurén (Eds): Languages for Special Purposes. An International Handbook, p. 548-562.

Schiffauer, Peter / Jdrzejowska-Schiffauer, Izabela (2019): Triumph of Law over Language. Case Studies on Multilingually Negotiated EU-Law, in: Friedemann Vogel (Ed): Legal Linguistics Beyond Borders: Language and Law in a World of Media, Globalisation and Social Conflicts. Relaunching the International Language and Law Association (ILLA), p. 187-206.

Simonnæs, Ingrid / Kristiansen, Marita (Eds) (2019): Legal translation. Current issues and challenges in research, methods and applications, Berlin.

Stoffel, Marina (2019): Mehrsprachige Gesetzesredaktion. Bedeutung des Übersetzungsprozesses in der Gesetzgebung mit Blick auf die Verfassungsrevision im Wallis, Masterarbeit, ZHAW, Dep. f. Angewandte Linguistik.

\subsection{Einzelfragen}

Agarwal, Anurag K. (2019): Legal language and business communication, Singapore.

Aviolat, Madeleine (2019): Erarbeiten der schweizerischen Rechtsterminologie in den vier Landessprachen und Englisch, in: Parlament der Deutschsprachigen Gemeinschaft Belgiens (Hrsg.): Nationale Variation in der deutschen Rechtsterminologie. Beiträge zur Tagung vom 12. und 13. Oktober 2018 im Seminarzentrum Kloster Heidelberg in Eupen, Eupen, S. 57-76.

Baumann, Antje (2019): DER Mensch, DIE Verwaltung und DAS Geschlecht. Zum Unterschied zwischen mitgemeint, mitgesagt und gleichberechtigt, in: LeGes 30 (2019) 1.

Bich-Carrière, Laurence (2019): Say it with [A Smiling Face with Smiling Eyes]: Judicial Use and Legal Challenges with Emoji Interpretation in Canada, in: International Journal for the Semiotics of Law 32/2, p. 283-319.

Breeze, Ruth (2019): Part-of-speech patterns in legal genres. Text-internal dynamics from a corpus-based perspective, in: Teresa Fanego / Paula Rodríguez-Puente (Eds) (2019): Corpusbased Research on Variation in English Legal Discourse, Amsterdam/Philadelphia, pp. 79-103.

Bruno, Giovanni (2019): L'aggettivo «sabatale»: un neologismo incipiente nel linguaggio burocratico-amministrativo?, in: LeGes 30 (2019) 3.

Conley, John M. / O’Barr, William M. / Conley Riner, Robin (Eds) (2019): Just Words. Law, Language, and Power, $3^{\text {rd }}$ ed., Chicago.

Fanego, Teresa / Rodríguez-Puente, Paula (Eds) (2019): Corpus-based Research on Variation in English Legal Discourse, Amsterdam/Philadelphia.

Hamann, Hanjo / Hürlimann, Daniel (Hrsg.) (2019): Open Access in der Rechtswissenschaft, Baden-Baden. 
Rebekka Bratschi / Astrid Hirzel / Céline Mavrot / Markus Nussbaumer / Lucas Stutz / Felix Uhlmann, Literaturhinweise - Repères bibliographiques - Riferimenti bibliografici - Indicaziuns bibliograficas, in: LeGes 31 (2020) 1

Kotthoff, Helga / Nübling, Damaris (2018): Genderlinguistik. Eine Einführung in Sprache, Gespräch und Geschlecht. Unter Mitarbeit von Claudia Schmidt, Tübingen.

Marazzini, Claudio (2018): La lingua, il giudice, la Costituzione. Una vertenza tutta italiana e un confronto internazionale, Ospedaletto.

Mostŕn, Martin (2018): Asylbewerber, Flüchtling oder Migrant? Zum Gebrauch von Personenbezeichnungen im institutionellen Diskurs zur Flüchtlingshtematik, in: Lenka Vaňková (Hrsg.): Fachlichkeit und Fachsprachlichkeit in varianten Kontexten, Berlin, S. 49-109.

Nicoletti, Antonella (2019): Der Rat für Deutschsprachige Terminologie und die Sektion Terminologie der Zentralen Sprachdienste der Schweizerischen Bundeskanzlei - ein Gründer, zwei Jubiläen, in: Petra Drewer / Donatella Pulitano (Hrsg.): Terminologie: Epochen - Schwerpunkte Umsetzungen. Zum 25-jährigen Bestehen des Rats für Deutschsprachige Terminologie, S. 155-165.

Nübling, Damaris (2018): Und ob das Genus mit dem Sexus. Genus verweist nicht nur auf Geschlecht, sondern auch auf die Geschlechterordnung, in: Sprachreport 34/3, S. 44-50.

Pulitano, Donatella (2019): Terminologiearbeit: im öffentlichen Dienst - im Dienste der Öffentlichkeit, in: Petra Drewer / Donatella Pulitano (Hrsg.): Terminologie: Epochen - Schwerpunkte - Umsetzungen. Zum 25-jährigen Bestehen des Rats für Deutschsprachige Terminologie, S. 143-153.

Schweighofer, Erich / Kummer, Franz / Saarenpää, Ahti (Hrsg.) (2019): Internet of Things. Tagungsband des 22. Internationalen Rechtsinformatik-Symposions, IRIS 2019, Proceedings of the 22nd International Legal Informatics Symposium, Bern.

Vogel, Friedemann / Bäumer, Benjamin / Deus, Fabian / Rüdiger, Jan Oliver / Tripps, Felix (2019): Die Bedeutung des Adjektivs «geschäftsmässig»im juristischen Fach- und massenmedialen Gemeinsprachgebrauch, in: LeGes 30 (2019) 3.

Wissik, Tanja / Resch, Claudia (2019): Rechtssprachliche Aspekte in Historischen Flugblättern des 18. Jahrhunderts, in: Fachsprache 41/1-2, S. 41-60.

Zangger, Alfred (2019): Nationale Varianten in abgestimmten deutschen Übersetzungen multilateraler Übereinkommen eine besondere Herausforderung für die Schweiz, in: Parlament der Deutschsprachigen Gemeinschaft Belgiens (Hrsg.): Nationale Variation in der deutschen Rechtsterminologie. Beiträge zur Tagung vom 12. und 13. Oktober 2018 im Seminarzentrum Kloster Heidelberg in Eupen, Eupen, S. 135-150.

Zuliani, Dario (2018): Concordanze lessicali italiane e francesi del Codice Napoleone, Firenze.

\subsection{Hilfsmittel}

Auswärtiges Amt (2018): Die amtlichen Bezeichnungen des Bundespräsidenten und der Bundesregierung - Deutsch, Berlin.

Conte, Giuseppe / Boss, Hans / Vollmer, Leonhard (2019): Wörterbuch der Rechts- und Wirtschaftssprache. Teil 1: Italienisch-Deutsch, 6. Aufl., Basel. 
Rebekka Bratschi / Astrid Hirzel / Céline Mavrot / Markus Nussbaumer / Lucas Stutz / Felix Uhlmann, Literaturhinweise - Repères bibliographiques - Riferimenti bibliografici - Indicaziuns bibliograficas, in: LeGes 31 (2020) 1

Dax, Peer / Hopf, Gerhard (Hrsg.) (2019): Abkürzungs- und Zitierregeln der österreichischen Rechtssprache und europarechtlicher Rechtsquellen (AZR), 8. Aufl., Wien.

Federal Chancellery (2019): Style Guide. A Handbook for Authors and Translators in the Federal Administration, Bern.

Ghisolfi, Beppe (2019): Lessico finanziario, Torino.

Intergruppo Italianità (2019): L'italiano in Parlamento. Vademecum, Berna.

Lenoble-Pinson, Michèle (2019): Dire et écrire le droit en français correct, au plaisir des gens de robe, 2. Aufl., Bruxelles.

Meier, Niklaus (2019): Terminologie juridique allemande, $3^{\mathrm{e}}$ éd. Bâle/Neuchâtel.

Piska, Christian M. (Hrsg.) (2019): Fachwörterbuch Rechtswissenschaften, 2. Aufl., Wien.

Ralli, Natascia / Andreatta, Norbert (2018): bistro - ein Tool für mehrsprachige Rechtsterminologie, in: trans-kom 11, S. 7-44.

Schiannini, Donata (2018): Come lo scrivo? Guida pratica a una lingua che cambia, Milano.

Weber, Klaus (Hrsg.) (2019): Rechtswörterbuch, 23. Aufl., München.

Zoppetti, Antonio (2018): L'etichettario. Dizionario di alternative italiane a 1800 parole inglesi, Firenze.

\section{Evaluation}

\subsection{Evaluationsansätze und -methoden}

Chouinard, Jill Anne / Bourgeois, Isabelle / Amo, Courtney (Eds) (2019): Growing the Knowledge Base in Evaluation: The Contributions of J. Bradley Cousins. Charlotte, NC.

King, Jean A. / Almin, Marvin C. (2019): The Centrality of Use: Theories of Evaluation Use and Influence and Thoughts on the First 50 Years of Use Research, in: American Journal of Evaluation, $40,3,431-458$.

Mavrot, Céline / Hadorn, Susanne / Sager, Fritz (2019): Mapping the Mix: Linking Instruments, Settings and Target Groups in the Study of Policy Mixes, in: Research Policy, 48, 10, 103614.

Patton, Michael Quinn (2019): Transformation to Global Sustainability: Implications for Evaluation and Evaluators, in: New Directions for Evaluation, 162, 103-117.

Pattyn, Valérie / Molenveld, Astrid / Befani, Barbara (2019): Qualitative Comparative Analysis as an Evaluation Tool: Lessons From an Application in Development Cooperation, in: American Journal of Evaluation, 40, 1, 55-74.

Ravn, Rasmus (2019): Testing mechanisms in large-N realistic evaluations, in: Evaluation, 25, 2, $171-188$. 
Rebekka Bratschi / Astrid Hirzel / Céline Mavrot / Markus Nussbaumer / Lucas Stutz / Felix Uhlmann, Literaturhinweise - Repères bibliographiques - Riferimenti bibliografici - Indicaziuns bibliograficas, in: LeGes 31 (2020) 1

Rolfe, Steve (2019): Combining Theories of Change and Realist Evaluation in Practice: Lessons from a Research on Evaluation Study, in: Evaluation, 25, 3, 294-316.

\subsection{Evaluationspraxis}

DöRING, Nicola (2019): Evaluationsforschung, in Nina Baur / Jörg Blasius (Hrsg.), Handbuch Methoden der empirischen Sozialforschung. Wiesbaden, 173-189.

Freund, Megan / Zucca, Alison / Sanson-Fisher, Robert / Milat, Andrew / Mackenzie, Lisa / Turon, Heidi (2019): Barriers to the Evaluation of Evidence-based Public Health Policy, in: Journal of Public Health Policy 40, 1, 114-125.

Perrin, Burt (2019): How to Manage Pressure to Change Reports: Should Evaluators Be Above Criticism? In: American Journal of Evaluation, 40, 3, 354-375.

Picciotto, Robert (2019): Is evaluation obsolete in a post-truth world?, in: Evaluation and Program Planning, 73, 88-96.

Rogers, Alison / Kelly, Leanne M. / McCoy, Alicia (2019): Evaluation Literacy: Perspectives of Internal Evaluators in Non-Government Organizations, in: The Canadian Journal of Program Evaluation, 34, 1, 1-20.

\subsection{Evaluation in Politik und Verwaltung}

Dahler-Larsen, Peter / Boodhoo, Adillah (2019), Evaluation culture and good governance: Is there a link? In: Evaluation, 25, 3, 277-293.

Dolder, Olivier (2019), Wie beeinflussen sich Evaluation und New Public Management? Analyse am Beispiel von Schweizer Kantonsverwaltungen, Bern.

Eberli, Daniela (2019): Die Nutzung von Evaluationen in den Schweizer Parlamenten, Zürich.

Hoerner, Julian M. (2019): Adding Fuel to the Flames? Politicisation of EU Policy Evaluation in National Parliaments, in: Politische Vierteljahresszeitschrift, 60, 4, 805-821.

Horber-Papazian, Katia / Baud-Lavigne, Marion (2019): Factors Contributing to the Strong Institutionalization of Policy Evaluation in Switzerland, in: Andreas Ladner / Nils Soguel / Yves Emery / Sophie Weerts / Stéphane Nahrath (Eds), Swiss Public Administration. Governance and Public Management. Cham, 355-371.

Horber-Papazian, Katia / Rosser, Christian (2019): From Law to Reality - A Critical View on The Institutionalization of Evaluation in The Swiss Canton of Geneva's Parliament, in : Jan-Eric Furubo / Nicoletta Stame (Hrsg.), The Evaluation Enterprise: A Critical View. New York, 83-110.

Mbav Yav, Germain (2019): Vers le «législateur évaluateur»?, Nécessité de surveiller l'exécution et les effets des lois en République démocratique du Congo, Lausanne. 
Rebekka Bratschi / Astrid Hirzel / Céline Mavrot / Markus Nussbaumer / Lucas Stutz / Felix Uhlmann, Literaturhinweise - Repères bibliographiques - Riferimenti bibliografici - Indicaziuns bibliograficas, in:

LeGes 31 (2020) 1

Nотвонм, RовеRт (2019): Wirkungen und Grenzen von Institutionen exekutiver Folgenabschätzungskontrolle - Nationaler Normenkontrollrat und Ausschuss für Regulierungskontrolle im Vergleich, Berlin.

Pattyn, Valérie / De Peuter, Bart / Brans, Marleen (2019): Why do Ministers Ask for Policy Evaluation Studies? The Case of the Flemish Government, in: Politische Vierteljahresschrift, 60, $4,701-717$.

Pотluка, Ото (2019): Overcoming Political Resistance to Evaluating the Environmental Impacts of EU Cohesion Policy, in: Politische Vierteljahresschrift, 60, 763-784.

Schoenefeld, Jonas J. / Jordan, Andrew J. (2019): Environmental Policy Evaluation in the EU: Between Learning, Accountability, and Political Opportunities?, in: Environmental Politics, 28, 2, 365-384.

Stephenson, Paul J. / Schoenefeld, Jonas J. / Leeuw, Frans L. (2019): The Politicisation of Evaluation: Constructing and Contesting EU Policy Performance, in: Politische Vierteljahresschrift, $60,4,663-679$.

\subsection{Evaluationsstudien}

Beywl, Wolfgang (2019): Evaluationskultur im Bildungsbereich, in: LeGes 30 (2019) 1.

Fässler, Sarah / Studer, Sibylle (2018): Wirkungsevaluation von Interventionen. Leitfaden für Projekte im Bereich Bewegung, Ernährung und psychische Gesundheit. Arbeitspapier 46. Bern / Lausanne.

Pelucha, Martin / Kveton, Viktor / Potluka, Оto (2019): Using Mixed Method Approach in Measuring Effects of Training in Firms: Case Study of the European Social Fund support, in: Evaluation and Program Planning, 73, 146-155.

Ritz, Manuel / Neumann, Oliver / Sager, Fritz (2019): Senkt New Public Management die Verwaltungsausgaben in den Schweizer Kantonen? Eine empirische Analyse über zwei Dekaden, in: Swiss Political Science Review, 25, 3, 226-252.

\subsection{Evaluationsfachlichkeit}

Balzer, Lars (2019): Die DeGEval-Standards im Vergleich zu empirisch bestimmten Bedingungen erfolgreicher Evaluationsprojekte, in: Jan Ulrich Hense / Wolfgang Böttcher / Michael Kalman / Wolfgang Meyer (Hrsg.), Evaluation: Standards in unterschiedlichen Handlungsfeldern. Münster, 297-310.

Balzer, LaRs (2019): Die Evaluationspraxis im Licht der neuen SEVAL-Standards sowie empirisch bestimmter Bedingungen erfolgreicher Evaluationsprojekte, in: LeGes 30 (2019) 3.

Beywl, Wolfgang / Böttcher, Wolfgang / Hense Jan / Widmer Tomas (2019): Vorläufer, Entstehung und Weiterentwicklung der Standards für Evaluation, in: Jan Ulrich Hense / 
Rebekka Bratschi / Astrid Hirzel / Céline Mavrot / Markus Nussbaumer / Lucas Stutz / Felix Uhlmann, Literaturhinweise - Repères bibliographiques - Riferimenti bibliografici - Indicaziuns bibliograficas, in: LeGes $31(2020) 1$

Wolfgang Böttcher / Michael Kalman / Wolfgang Meyer (Hrsg.), Evaluation: Standards in unterschiedlichen Handlungsfeldern. Münster, 15-32.

Gullickson, Amy M. / King, Jean A. / LaVelle, John M. / Clinton, Janet M. (2019): The Current State of Evaluator Education: A Situation Analysis and Call to Action, in: Evaluation and Program Planning, 75, 20-30.

Rüefli, Christian / Zweidler, Reinhard (2019): Die Revision der SEVAL-Standards - ein Erfahrungsbericht, in: Jan Ulrich Hense / Wolfgang Böttcher / Michael Kalman / Wolfgang Meyer (Hrsg.), Evaluation: Standards in unterschiedlichen Handlungsfeldern. Münster, 261-276. 